\title{
PRODUCTION OF AN ACCELERATED OXYGEN-14 BEAM*
}

\author{
J. Powell ${ }^{\dagger}$, J. P. O’Neil and Joseph Cerny \\ Lawrence Berkeley National Laboratory, Berkeley, CA 94720, U.S.A.
}

BEARS is an ongoing project to provide a light-ion radioactive-beam capability at the 88-Inch Cyclotron at LBNL. Light radioactive isotopes are produced at a $10 \mathrm{MeV}$ proton medical cyclotron, transported $350 \mathrm{~m}$ via a high-speed gas transport capillary, cryogenically separated, and injected into the 88-Inch Cyclotron's ion source. The first radioactive beam successfully accelerated was Carbon-11 and beams of intensity more than $10^{8}$ ions/sec have been utilized for experiments.

Development of Oxygen-14 as the second BEARS beam presented considerable technical challenges, both due to its short half-life of 71 seconds and the radiation chemistry of oxygen in the target. The usual techniques developed for medical uses of Oxygen-15 involve the addition of significant amounts of carrier oxygen, something that would overload the ion source.

As a solution, Oxygen-14 is produced as water in a carrier-free form, and is chemically converted in two steps to carbon dioxide, a form readily usable by the BEARS. This system has been built and is operational, and initial tests of accelerating an Oxygen-14 beam have been performed.

PACS: 29.25.Rm; 25.60.-t

Keywords: Radioactive beams; Oxygen-14 beam; BEARS

\footnotetext{
Work supported by the US Department of Energy, under contract DE-AC03-76SF00098.

† E-mail: jdpowell@LBL.GOV
} 
This paper describes the production of an accelerated Oxygen-14 beam at the 88Inch Cyclotron. This is the second beam developed with the systems of BEARS, or Berkeley Experiments with Accelerated Radioactive Species, the first being Carbon-11 [1]. The ${ }^{14} \mathrm{O}$ beam is intended for use in a number of experiments. Key among them is the astrophysical reaction ${ }^{14} \mathrm{O}(\alpha, \mathrm{p}){ }^{17} \mathrm{~F}$, important for understanding Novae explosions and $\mathrm{X}$-ray bursts [2]. Another experiment would attempt to measure two proton decay in ${ }^{14} \mathrm{O}(\alpha, 2 \mathrm{p})$, through a $1^{-}$resonance in ${ }^{18} \mathrm{Ne}$ that cannot decay sequentially. This decay has recently been observed in ${ }^{17} \mathrm{~F}(\mathrm{p}, 2 \mathrm{p})$ [3], although it was not yet possible to determine if the decay occurred via ${ }^{2} \mathrm{He}$ emission or simultaneous individual protons, and the observed decay rate is significantly higher than predicted [4]. Oxygen-14 provides an alternate route into the relevant state of ${ }^{18} \mathrm{Ne}$ that has a number of advantages, including avoidance of possible contributions from ${ }^{17} \mathrm{~F}$ breakup (to ${ }^{16} \mathrm{O}+\mathrm{p}$ ) on the hydrogencontaining target or from decay of a nearby state of unnatural parity in ${ }^{18} \mathrm{Ne}[4]$.

Production of the ${ }^{14} \mathrm{O}$ beam begins at LBNL's Biomedical Isotope Facility (BIF) using a $40 \mu \mathrm{A}, 10 \mathrm{MeV}$ proton beam bombarding high-pressure nitrogen gas targets. Both ${ }^{14} \mathrm{O}$ and ${ }^{11} \mathrm{C}$ are produced in the same nitrogen target, through the ${ }^{14} \mathrm{~N}(\mathrm{p}, \mathrm{n})$ and $(\mathrm{p}, \alpha)$ reactions, respectively. BIF is located about $350 \mathrm{~m}$ from the 88 -Inch Cyclotron, and activity is rapidly transported as carbon dioxide in a flow of helium through a $3 \mathrm{~mm}$ I.D. tube that connects the two buildings. At the 88-Inch Cyclotron, the activity is cryogenically separated from the bulk of the carrier helium, before being injected into an ECR ion source for subsequent ionization and acceleration. Details of BEARS, and its use for the production of ${ }^{11} \mathrm{C}$ beams, may be found in Reference 1. Development of this 
${ }^{14} \mathrm{O}$ work was aided by the extensive literature on the production, for PET studies, of Oxygen-15 via similar reactions on nitrogen targets (such as ${ }^{14} \mathrm{~N}(\mathrm{~d}, \mathrm{n}){ }^{15} \mathrm{O}$ ).

Isotope production at BIF is complicated by radiolysis and target-chamber surface effects. The intensely ionizing radiation fields present in the $8 \mathrm{ml}$ target during bombardment are sufficient to break up most of the original molecules into atoms and radicals [5]. This both chemically processes the material and causes activity loss through reactions with the target walls. With ${ }^{11} \mathrm{C}$, it was found that much of the activity was lost on the walls unless sufficient oxygen, about $0.2 \%$, was added to the target nitrogen gas to radiolytically process the material to $\mathrm{CO}_{2}[1]$.

Initial tests at the 88-Inch Cyclotron, performed with large-volume targets and low proton beam intensities $(<1 \mu \mathrm{A})$, had succeeded in producing significant yields of ${ }^{14} \mathrm{O}$ in a suitable chemical form for BEARS (perhaps $\mathrm{NO}$ or $\mathrm{N}_{2} \mathrm{O}$ ). However, these results could not be repeated with the small BIF target and full production beam intensity. All the ${ }^{14} \mathrm{O}$ appeared to be lost on the target walls, and very little ${ }^{14} \mathrm{O}$ was recovered. This is consistent with work with ${ }^{15} \mathrm{O}$, where significant yields of $\mathrm{N}^{15} \mathrm{O}$ have been observed with a very large target [6], while no $\mathrm{N}^{15} \mathrm{O}$ and only quite small yields of $\mathrm{N}_{2}{ }^{15} \mathrm{O}$ are found in smaller target studies [7].

To recover the ${ }^{14} \mathrm{O}$ from the target walls requires the addition of a suitable additive that will react strongly with oxygen. In ${ }^{15} \mathrm{O}$ work it is common to add oxygen or carbon dioxide [8]; carbon dioxide in particular would produce ${ }^{14} \mathrm{O}$ directly as $\left[{ }^{14} \mathrm{O}\right] \mathrm{CO}_{2}$. Unfortunately, this entails the dilution of the ${ }^{14} \mathrm{O}$ in a large amount of regular oxygen. The amount of additive needed to prevent loss on the walls, generally a fraction of a percent or more of the target gas, is at least two orders of magnitude more gas load than 
the 88-Inch's ion source can handle. Because of radiolytic processing, any additive containing oxygen will cause irreparable dilution of the activity ${ }^{1}$.

Thus, a mixture of a few percent hydrogen in nitrogen was used to produce ${ }^{14} \mathrm{O}$ in the form of water, $\mathrm{H}_{2}{ }^{14} \mathrm{O}$, avoiding the addition of any oxygen. As with the use of oxygen for ${ }^{11} \mathrm{C}$ production, it is likely that the hydrogen serves to recover ${ }^{14} \mathrm{O}$ atoms from the walls in addition to reacting in the gas phase. Roughly half of the calculated thicktarget production of ${ }^{14} \mathrm{O}$ was recovered as water, a similar fraction to that of the ${ }^{11} \mathrm{CO}_{2}$ that can be produced in the same target.

The aluminum BIF target chamber used for $\mathrm{H}_{2}{ }^{14} \mathrm{O}$ production was gold-plated as a precaution against possible isotopic exchange on aluminum oxide surfaces. Two or three layers of $\mathrm{Al}_{2} \mathrm{O}_{3}$ are known to isotopically exchange with oxygen in the gas phase at temperatures of around $500^{\circ} \mathrm{C}$ [9], and radiolysis could cause similar effects. When a non-gold-plated aluminum target was briefly tried, it yielded very little recoverable $\mathrm{H}_{2}{ }^{14} \mathrm{O}$.

Unfortunately, water is not a good chemical form for use with BEARS, since it can stick in the long $(350 \mathrm{~m})$ unheated transport lines. To overcome this problem, we have constructed an automated system to convert the water to carbon dioxide. This is illustrated in Figure 1.

First, the target is unloaded and the water temporarily frozen in a small coil of 1.6 mm o.d., $1.0 \mathrm{~mm}$ i.d. stainless steel tubing, cooled to $-40^{\circ} \mathrm{C}$ by a stream of cold dry air

\footnotetext{
${ }^{1}$ An attempt to produce $\left[{ }^{14} \mathrm{O}\right] \mathrm{CO}_{2}$ through reaction with added carbon monoxide, with the intention of later cryogenic separation, failed because $\mathrm{CO}$ is radiolytic processed to $\mathrm{CO}_{2}+\mathrm{C}_{3} \mathrm{O}_{2}$ [5].
} 
from a vortex tube ${ }^{2}$. This allows the elimination of the other target-gas components, producing a clean sample of $\mathrm{H}_{2}{ }^{14} \mathrm{O}$. The other components include large amounts of radiolytically-produced ammonia along with the original hydrogen, both of which can complicate further steps. Co-produced ${ }^{11} \mathrm{CH}_{4}$ is also separated, reducing radiation issues $^{3}$. It is hoped that this separation step will eventually allow development of a recirculated Nitrogen-15 target, allowing production of an Oxygen-15 beam (through $\left.{ }^{15} \mathrm{~N}(\mathrm{p}, \mathrm{n}){ }^{15} \mathrm{O}\right)$ with the same system.

The ${ }^{14} \mathrm{O}$-water is released through momentary resistive heating of the steel trapping coil with a strong electric current ${ }^{4}$. A flow of helium carries the activity through a small tube furnace containing carbon granules at high temperatures $\left(1000-1100^{\circ} \mathrm{C}\right)$. This converts the water vapor to carbon monoxide $\left(\mathrm{H}_{2}{ }^{14} \mathrm{O}+\right.$ carbon $\left.\Rightarrow \mathrm{C}^{14} \mathrm{O}+\mathrm{H}_{2}\right)$. The carbon monoxide is then oxidized to carbon dioxide over a platinum catalyst at $180^{\circ} \mathrm{C}$, with a small amount of oxygen added to the helium flow for this purpose. The resulting $\left[{ }^{14} \mathrm{O}\right] \mathrm{CO}_{2}$ is then in a chemical form used by the existing BEARS system, currently in use for ${ }^{11} \mathrm{CO}_{2}$.

Owing to the limited mass of processed material, it was found to be necessary to limit the surface area of both the carbon and the oxidation catalyst used. Activated carbon (with areas of many square meters per gram) seemed to retain most or all of the ${ }^{14} \mathrm{O}$, even at $1100^{\circ} \mathrm{C}$, while commercial platinum catalysts, consisting of platinum deposited on high-surface-area alumina beads, retarded and spread out the appearance of

\footnotetext{
${ }^{2}$ Model 106, ITW Vortec, Cincinnati, OH.

${ }^{3} \mathrm{The}^{11} \mathrm{CH}_{4}$ is oxidized at $700^{\circ} \mathrm{C}$ over a $\mathrm{CuO}$ catalyst and captured on Ascarite.

${ }^{4}$ About 15 A for three seconds.
} 
$\left[{ }^{14} \mathrm{O}\right] \mathrm{CO}_{2}$ over more than a minute ${ }^{5}$. The carbon finally used was a simple graphite rod $^{6}$, broken into a few pieces of about $6 \mathrm{~mm}$ in diameter, while the catalyst was a few square centimeters of platinum foil. With these low-surface-area materials, the complete conversion process takes about 10 seconds, and conversion efficiency is better than $50 \%$.

With ${ }^{14} \mathrm{O}$, which has a halflife of 71 seconds, the BEARS system is operated on a cycle of about 100 seconds, faster than the 5 minute cycle used with ${ }^{11} \mathrm{C}$. The BIF cyclotron is used to provide $40 \mu \mathrm{A}$ of $10 \mathrm{MeV}$ protons for periods of $90 \mathrm{sec}$, with ten seconds for loading and unloading the target with the nitrogen plus hydrogen mix. After unloading and trapping the $\mathrm{H}_{2}{ }^{14} \mathrm{O}$, the trapping coil is briefly flushed to waste to eliminate any remaining target gas. Then the helium flow is directed through the chemical conversion system as the coil is quickly heated. Conversion takes about ten seconds, and the $\left[{ }^{14} \mathrm{O}\right] \mathrm{CO}_{2}$ is collected in a small holding tank. From this point on the process is generally as described in Ref 1 . The activity is rapidly transported $350 \mathrm{~m}$ to the 88-Inch Cyclotron by being driven down a pre-evacuated $3 \mathrm{~mm}$ i.d. tube with 6 atm of helium. Originally, BEARS used nitrogen as a drive gas, but helium allows for shorter transport times, 12 versus 22 seconds. The cryogenic trap that separates the activity from the gas stream currently can be heated enough to release carbon dioxide in about $20 \mathrm{sec}$, although this may be improved in the future. The released $\left[{ }^{14} \mathrm{O}\right] \mathrm{CO}_{2}$ is temporarily held in a $40 \mathrm{~cm}^{3}$ reservoir while it is slowly bled into the ion source at a controlled rate. This source, the AECR-U, has in the past been measured to have an efficiency of $3.6 \%$ for ionization of ${ }^{14} \mathrm{O}$ to the $6+$ charge state [1].

\footnotetext{
${ }^{5}$ Attempts at using $\mathrm{CuO}$ as an oxidation catalyst failed due to complete ${ }^{14} \mathrm{O}$ retention, most likely due to isotopic exchange [9].

${ }^{6}$ AGKSP grade, Ultra "F" purity, Stk \# 4076, Alfa Aesar, Ward Hill, MA
} 
We are in the early stages of testing the acceleration of the ${ }^{14} \mathrm{O}^{6+}$ beam through the 88-Inch Cyclotron. Initial tests produced a non-optimized on-target intensity of about $10^{5}$ ions/per second. This was without the necessary diagnostic ability to tune either the ion source or the cyclotron on the ${ }^{14} \mathrm{O}$ beam itself; instead the accelerator was pre-tuned with ${ }^{14} \mathrm{~N}^{6+}$. Potential intensity of the ${ }^{14} \mathrm{O}$ beam can be estimated from our past work with ${ }^{11} \mathrm{C}$ beams, which have better than $1 \times 10^{8}$ ions/sec on target. By adjusting for the lower thick-target yield $\left(5 \times 10^{9}\right.$ for ${ }^{14} \mathrm{O}$ versus $1 \times 10^{11}$ atoms/sec for $\left.{ }^{11} \mathrm{C}[10]\right)$, the chemical conversion efficiency, the differing half-life losses, and the lower ionization efficiency measured for ${ }^{14} \mathrm{O}$, we estimate an ${ }^{14} \mathrm{O}$ beam intensity on target of at least $5 \times 10^{5}$ ions/sec. If future development of a recirculating ${ }^{15} \mathrm{~N}$ target permits an ${ }^{15} \mathrm{O}$ beam with this system, then, given the twenty times higher thick-target yield [10] and the longer halflife of ${ }^{15} \mathrm{O}$, we estimate a potential ${ }^{15} \mathrm{O}$ beam intensity of $1.5 \times 10^{7}$ ions/sec.

\section{References}

[1] J. Powell et al., Nucl. Instr. and Meth. A 455 (2000) 452.

[2] D. W. Bardayan and M. S. Smith, Phys. Rev. C 56 (1997) 1647.

[3] J.Gomez del Campo et al., Phys.Rev.Lett. 86 (2001) 43.

[4] L. V. Grigorenko et al., Phys. Rev. C 65 (2002) 044612.

[5] P. Ausloos (Ed.): Fundamental Processes in Radiation Chemistry, Wiley, New York, 1968.

[6] K. A. Krohn, J. M. Link and W. G. Weitkamp, Radiochim. Acta 88 (2000) 193.

[7] S. C. Van Der Linde et al., Appl. Radiat. Isot. 52 (2000) 77. 
[8] R. A. Ferrieri and A. P. Wolf, Radiochim. Acta 34 (1983) 69.

[9] A. Bielansk and J. Haber, Oxygen in Catalysis, Marcel Dekker Inc., New York, 1991, p. $114-121$.

[10] S. wa Kitwanga et al., Phys. Rev. C 42 (1990) 748. 


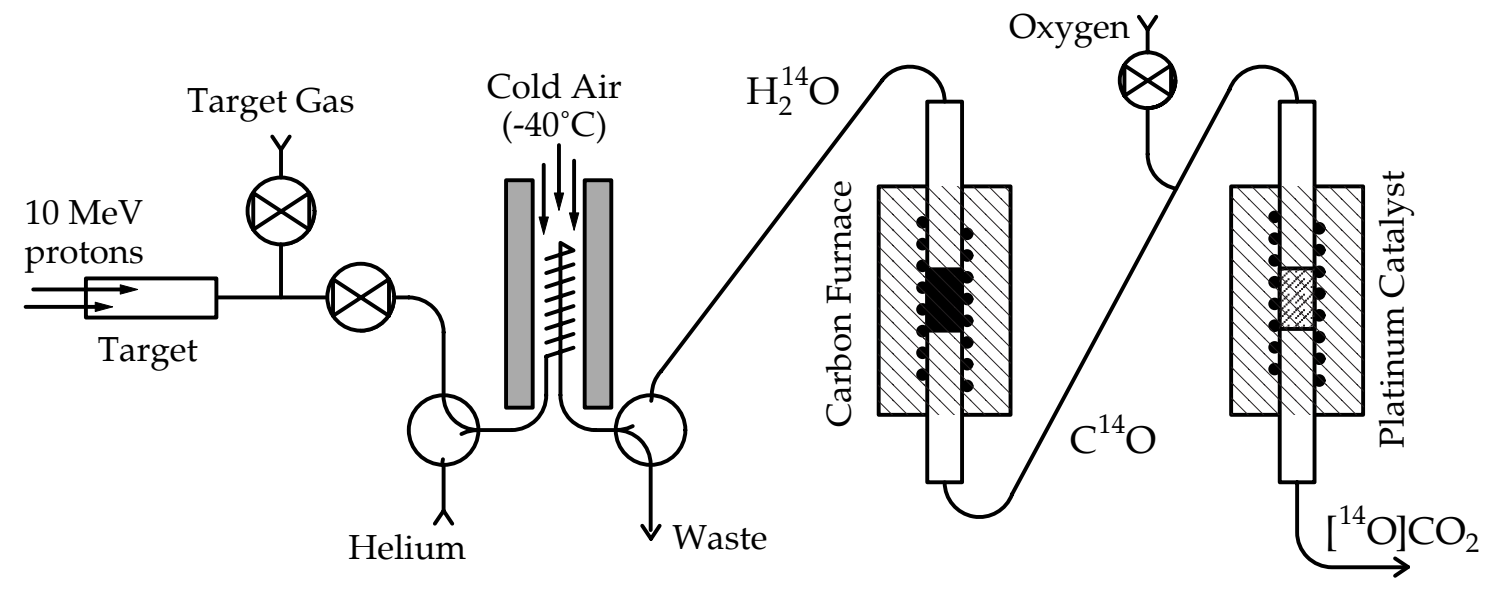

Figure 1. System for production of $\mathrm{H}_{2}{ }^{14} \mathrm{O}$ and conversion to $\left[{ }^{14} \mathrm{O}\right] \mathrm{CO}_{2}$. Target gas is unloaded to waste through a small coil of stainless steel tubing kept cold by a flow of $40^{\circ} \mathrm{C}$ air from a vortex tube, in order to freeze out and separate the $\mathrm{H}_{2}{ }^{14} \mathrm{O}$. The activity, released by applying a strong electric current to resistively heat the coil, is carried in a flow of helium through two chemical conversion steps. The first converts $\mathrm{H}_{2}{ }^{14} \mathrm{O}$ to $\mathrm{C}^{14} \mathrm{O}$ over graphite at $1000-1100^{\circ} \mathrm{C}$; the second oxidizes the carbon monoxide to $\left[{ }^{14} \mathrm{O}\right] \mathrm{CO}_{2}$ over platinum at $180^{\circ} \mathrm{C}$. 